\title{
Drilling short geodesics in hyperbolic 3-manifolds
}

\author{
K. Bromberg ${ }^{1}$
}

\begin{abstract}
We give an expository account of the deformation theory of geometrically finite, 3-dimensional hyperbolic cone-manifolds and its application to three classical conjectures about Kleinian groups.
\end{abstract}

\section{Introduction}

In a series of papers ([HK98, HK02, HK]), Hodgson and Kerckhoff developed a deformation theory for 3-dimensional hyperbolic cone-manifolds which they used to prove various important results about closed and finite volume hyperbolic 3-manifolds. This deformation theory was extended to infinite volume, geometrically finite hyperbolic cone-manifolds in [Br2, Br04]. In this setting the deformation theory has had a number of applications to classical conjectures about Kleinian groups.

Here is an example of a basic problem that can be addressed via the deformation theory. Let $(M, g)$ be a geometrically finite hyperbolic 3-manifold that contains a simple closed geodesic $\gamma$. Let $\hat{M}=M \backslash \gamma$ be the complement of $\gamma$. There will be then be a unique, geometrically finite, complete hyperbolic metric $\hat{g}$ on $\hat{M}$ such that the conformal boundaries of $(M, g)$ and $(\hat{M}, \hat{g})$ agree. We have the following theorem

Theorem 1.1 ([BB04]). For each $K>1$ there exists an $\ell>0$ such that if the length of $\gamma$ in $(M, g)$ is less then $\ell$ then there exists a $K$-bi-Lipschitz map

$$
\phi:(M \backslash \mathbb{T}, g) \longrightarrow(\hat{M} \backslash \hat{\mathbb{T}}, \hat{g})
$$

where $\mathbb{T}$ and $\hat{\mathbb{T}}$ are Margulis tubes about $\gamma$ and the rank two cusp, respectively.

We call such a theorem a "drilling theorem" for we have drilled the geodesic $\gamma$ out of the hyperbolic manifold $(M, g)$.

The way we obtain geometric control of the metric $\hat{g}$ is to interpolate between $g$ and $\hat{g}$ using hyperbolic cone-metrics. The Hodgson-Kerckhoff deformation theory gives means to bound the change in geometry as this one-parameter family of metrics varies. The first part of this paper will be an exposition of this deformation theory emphasizing the most geometric parts. For an expository account of Hodgson and

\footnotetext{
${ }^{1}$ Supported by a grant from the NSF
} 
Kerckhoff's work see [HK03]. To keep this paper somewhat self-contained there is some necessary overlap between the two papers.

In the second part of the paper we will apply the deformation theory to a collection of classical conjectures in Kleinian groups: the density conjecture, density of cusps on the boundary of quasiconformal deformation spaces and the ending lamination conjecture. Rather than discussing these conjectures in their full generality we will restrict to the special case of a Bers' slice. This will allow us to demonstrate how the deformation theory plays a role in approaching the conjectures in a simpler setting.

Acknowledgments. This paper is an expanded version of a talk given at the workshop on Spaces of Kleinian groups and Hyperbolic 3-manifolds held at the Newtown Institute in August 2003. The author would like to thank Caroline Series, Yair Minsky and Makoto Sakuma for organizing the workshop and their solicitation of this article.

The author would also like to thank his collaborator, Jeff Brock, with whom he did much of the work described in this paper.

\section{Deformations of hyperbolic metrics}

We will begin by examing the various different ways one can study a family of hyperbolic metrics: as Riemannian metrics, as $(G, X)$-structures and as representations of the fundamental group in the space of hyperbolic isometries. We will see the advantages of each viewpoint and the connections between the different viewpoints. A reference for this material is $\S 1$ and $\S 2$ of [HK98].

In the final subsection we will discuss complex projective structures on surfaces. These arise naturally as the boundary of hyperbolic 3-manifolds and will play an important role in the extension of the Hodgson-Kerckhoff deformation theory to infinite volume and geometrically finite hyperbolic cone-manifolds.

\subsection{One-parameter families of metrics}

We start with a family of metrics, $g_{t}: V \times V \longrightarrow R$, on a finite dimensional vector space $V$. For each $t$ there is a unique $\eta_{t} \in \operatorname{hom}(V, V)$ such that

$$
\frac{d g_{t}(v, w)}{d t}=2 g_{t}\left(v, \eta_{t}(w)\right)
$$

Since $g_{t}$ is symmetric, $\eta_{t}$ is self-adjoint, i.e.

$$
g_{t}\left(\eta_{t}(v), w\right)=g_{t}\left(v, \eta_{t}(w)\right) .
$$

We measure the size of $\eta_{t}$ using the metric $g_{t}$. Let $\left\{e_{1}, \ldots, e_{n}\right\}$ be an orthonormal basis for $V$ in the $g_{t}$ metric. Then define the norm of $\eta_{t}$ by the formula

$$
\left\|\eta_{t}\right\|^{2}=\sum g_{t}\left(\eta_{t}\left(e_{i}\right), \eta_{t}\left(e_{i}\right)\right) .
$$


For any $v, w \in V$ we then have

$$
g_{t}\left(v, \eta_{t}(w)\right) \leq 2\left\|\eta_{t}\right\| g_{t}(v, w) .
$$

By integrating (2.1) we see that if $\left\|\eta_{t}\right\| \leq K$ for all $t \in[0, T]$ then

$$
e^{-2 K T} g_{0}(v, v) \leq g_{T}(v, v) \leq e^{2 K T} g_{0}(v, v) .
$$

In particular the identity map on $V$ is a $K T$-bi-Lipschitz map from the $g_{0}$-metric to $g_{T}$-metric.

The trace of $\eta_{t}$ is the divergence and it is the derivative of the volume. The traceless part of $\eta_{t}$ is the strain and it measures the change in the conformal structure.

\subsection{Metrics on a manifold}

Now we apply the above work to a family of metrics, $g_{t}$, on a differentiable manifold $M$. In this setting $\eta_{t}$ is a one-parameter family in $\operatorname{hom}(T M, T M)$. Let $\left\|\eta_{t}(p)\right\|$ be the pointwise norm of $\eta_{t}$. Let $\phi_{t}:\left(M, g_{0}\right) \longrightarrow\left(M, g_{t}\right)$ be the identity map on $M$. If $\left\|\eta_{t}(p)\right\| \leq K$ for all $p \in M$ and all $t \in[0, T]$ then $\phi_{t}$ is a $K T$-bi-Lipschitz diffeomorphism.

The identity map on $M$ may not have the smallest bi-Lipschitz constant of all maps from $\left(M, g_{0}\right)$ to $\left(M, g_{t}\right)$. In particular for an arbitrary family of metrics there is no reason to hope that we can control the norm of $\eta_{t}$. The driving idea behind the HodgsonKerckhoff deformation theory is to find one-parameter families of hyperbolic metrics $g_{t}$ where the derivative $\eta_{t}$ is a harmonic strain field. As we will see below, this extra structure will allow us to control the norm of $\eta_{t}$.

\subsection{Hyperbolic metrics on a manifold}

Let $\mathscr{H}(M)$ be the space of all hyperbolic metrics on $M$. Two metrics $g$ and $h$ in $\mathscr{H}(M)$ are equivalent if there is a diffeomorphism $\psi: M \longrightarrow M$ isotopic to the identity such that $h=\psi^{*} g$. Given two equivalence classes of metrics we want to find an efficient path between them. That is we want to find a path $g_{t}$ that minimizes the derivative $\eta_{t}$. The last statement can be interpreted in a number of ways. For example, we could try to minimize the pointwise or $L^{2}$-norm of $\eta_{t}$. However, if $M$ is not compact then both of these norms can and will be infinite. Our efficient paths will have two properties. First, they will be divergence free so that $\eta_{t}$ is a strain field. Second they will be harmonic. We will not formally define harmonic. Informally, one can think of a harmonic strain field as locally minimizing the $L^{2}$-norm (see Appendix B of [Mc96]).

A harmonic strain field satisfies the following important equation:

Theorem 2.1. Let $(M, g)$ be a compact hyperbolic manifold with boundary and let $\eta$ be a harmonic strain field. Then

$$
\int_{M}\|\eta\|^{2}+\|\nabla \eta\|^{2}=\int_{\partial M} * \nabla \eta \wedge \eta
$$


This formula is very important because it allows us to compute the $L^{2}$-norm of a strain field by only knowing information on the boundary. We also note that $\eta$ is harmonic if it satisfies (2.3) for all compact submanifolds.

Another feature of harmonic strain fields is that they satisfy a mean value inequality:

Theorem 2.2. Let $(M, g)$ be a hyperbolic manifold and $\eta$ a harmonic strain field. If $B$ is a ball in $M$ of radius $R>\frac{\pi}{\sqrt{2}}$ centered at $p$ then

$$
\|\eta(p)\| \leq \frac{3 \sqrt{2(B)}}{4 \pi f(R)} \sqrt{\int_{B}\|\eta\|^{2} d V}
$$

where $f(R)=\cosh (R) \sin (\sqrt{2} R)-\sqrt{2} \sinh (R) \cos (\sqrt{2} r)$.

Together, Theorems 2.1 and 2.2 will allow us to get pointwise bounds on the the norm of $\eta$, at least for points in the thick part of $(M, g)$.

\subsection{Developing maps}

Another way to think of a hyperbolic structure is as a $(G, X)$-structure, where $X$ is hyperbolic space and $G$ the group of hyperbolic isometries. A $(G, X)$ structure is an atlas of charts to $X$ with transition maps which are restrictions of elements of $G$. A $(G, X)$-structure determines a developing map and a holonomy representation.

Here's how it works for a hyperbolic 3-manifold: A developing map is a local diffeomorphism,

$$
D: \tilde{M} \longrightarrow \mathbb{H}^{3},
$$

and the holonomy representation is a representation of the fundamental group,

$$
\rho: \pi_{1}(M) \longrightarrow P S L_{2} \mathbb{C}=\operatorname{Isom}^{+}\left(\mathbb{H}^{3}\right) .
$$

The developing map commutes with the action of the fundamental group where the fundamental groups acts on $\tilde{M}$ as deck transformations and on $\mathbb{H}^{3}$ via the holonomy representation. That is

$$
D(\gamma(x))=\rho(\gamma) D(x)
$$

for all $\gamma \in \pi_{1}(M)$. Let $\tilde{g}$ be the pull back of the hyperbolic metric. Then (2.4) implies that $\tilde{g}$ is equivariant and descends to a hyperbolic metric $g$ on $M$.

Conversely, a hyperbolic manifold, $(M, g)$, determines a developing map and holonomy representation. The developing map is unique up to post-composition with hyperbolic isometries. If we post-compose the developing with an isometry $\alpha \in P S L_{2} \mathbb{C}$ then we conjugate the holonomy by $\alpha$. 
Given a smooth family of hyperbolic metrics $\left(M, g_{t}\right)$, there is a smooth family of developing maps $D_{t}$, and holonomy representations $\rho_{t}$. The derivative of the developing maps determines a family of vector fields $v_{t}$ on $\tilde{M}$ in the following way. For a point $x \in \tilde{M}, D_{t}(x)$ is smooth path in $\mathbb{H}^{3}$. Let $v_{t}(x)$ be the pull-back, via $D_{t}$, of the tangent vector of this path at time $t$. These vector fields are not equivariant. However, they do satisfy the following automorphic property. For all $\gamma \in \pi_{1}(M)$ the difference, $\gamma_{*} v_{t}-v_{t}$, is an infinitesimal isometry in the $\tilde{g}_{t}$-metric. That is, the flow of the vector field $\gamma_{*} v_{t}-v_{t}$ is an isometry. This follows directly from differentiating (2.4).

The automorphic vector fields $v_{t}$, lead to the connection between the developing maps and the derivative, $\eta_{t}$, of the metrics $g_{t}$. The covariant derivative, $\nabla_{t} v_{t}$, is an element of hom $(T \tilde{M}, T \tilde{M})$. Let $\operatorname{sym} \nabla_{t} v_{t}$ be its symmetric part. The covariant derivative of an infinitesimal isometry is skew. Therefore, the automorphic property of $v_{t}$ implies that $\operatorname{sym} \nabla_{t} v_{t}$ is equivariant and descends to an element of hom $(T M, T M)$. By noting that the derivative $\frac{d g_{t}(v, w)}{d t}$ is the Lie derivative $\mathscr{L}_{v_{t}} g_{t}(v, w)$ we see that $\operatorname{sym} \nabla_{t} v_{t}=\eta_{t}$.

\subsection{Holonomy representations}

Let $\mathscr{R}(M)$ be the space of representations of $\pi_{1}(M)$ in $P S L_{2} \mathbb{C}$. We are only interested in representations up to conjugacy so we would like to study the quotient of $\mathscr{R}(M)$ under the action of $P S L_{2} \mathbb{C}$ by conjugacy. Unfortunately, this quotient may not be a nice object. For instance it may not even by Hausdorff. Instead one takes the Mumford quotient of $\mathscr{R}(M)$ which we denote $R(M)$. The Mumford quotient is an algebraic variety and its Zariski tangent space at a representation $\rho$ is the cohomology group $H^{1}\left(\pi_{1}(M) ; \operatorname{Ad} \rho\right)$. It will turn out, that at all points were are interested in, $R(M)$ is simply the topological quotient of $\mathscr{R}(M)$ by conjugacy. Furthermore, at these points $R(M)$ will be a differentiable manifold and the the Zariski tangent space will be naturally identified with the differentiable tangent space. For this reason we will ignore the distinction between the Mumford quotient and the topological quotient.

By differentiating a smooth family of representations $\rho_{t}$ we can see how the differentiable tangent space at each $\rho_{t}$ is identified with $H^{1}\left(\pi_{1}(M)\right.$; $\left.\operatorname{Ad} \rho_{t}\right)$. Let $\gamma$ be an element of $\pi_{1}(M)$. Then $\rho_{t}(\gamma)$ is a smooth path in $P S L_{2} \mathbb{C}$. Each tangent space of $P S L_{2} \mathbb{C}$ is canonically identified with the Lie algebra $s l_{2} \mathbb{C}$. Therefore the derivative $\dot{\rho}_{t}$ can be thought of as a map

$$
\dot{\rho}_{t}: \pi_{1}(M) \longrightarrow s l_{2} \mathbb{C}
$$

for each $t$. This map satisfies the cocyle condition

$$
\dot{\rho}_{t}(\gamma \beta)=\dot{\rho}_{t}(\gamma)+\operatorname{Ad} \rho_{t}(\gamma) \dot{\rho}_{t}(\beta)
$$

for all $\gamma$ and $\beta$ in $\pi_{1}(M)$ and therefore determines a cohomology class in $H^{1}\left(\pi_{1}(M)\right.$; $\left.\operatorname{Ad} \rho_{t}\right)$.

We also remark that $\dot{\rho}_{t}(\gamma)$ corresponds to the vector field $\gamma_{*} v_{t}-v_{t}$. The latter vector field is identified with an element of $s l_{2} \mathbb{C}$ by pushing forward $\gamma_{*} v_{t}-v_{t}$ via $D_{t}$. 
This push foward is an infinitesimal isometry on $\mathbb{H}^{3}$ and the space of infinitesimal isometries of $\mathbb{H}^{3}$ is canonically identified with $s l_{2} \mathbb{C}$.

\subsection{Complex projective structures}

A complex projective structure on a surface $S$ is an atlas of charts to the Riemann sphere, $\widehat{\mathbb{C}}$, where the transition maps are restrictions of elements of $P S L_{2} \mathbb{C}$. A projective structure is another example of $(G, X)$-structure where $G=P S L_{2} \mathbb{C}$ and $X=\widehat{\mathbb{C}}$. Let $P(S)$ be the space of projective structures on $S$. Since the action of $P S L_{2} \mathbb{C}$ is conformal, a projective structure also determines a conformal structure on $S$ so there is a map

$$
P(S) \longrightarrow T(S)
$$

where $T(S)$ is the Teichmüller space of marked conformal structures on $S$. One is often interested in the space of projective structures with a fixed conformal structure $X$. We denote the space of such structures $P(X)$.

Elements of $P S L_{2} \mathbb{C}$ take round circles in $\widehat{\mathbb{C}}$ to round circles. Therefore, there is a well defined notion of a round circle on a projective structure. A conformal map $f$ between two projective structures $\Sigma$ and $\Sigma^{\prime}$ will distort these round circles. The Schwarzian derivative, $S f$, measures this distortion. We will not give an exact definition of $S f$ although we will describe an infinitesimal version below. We will however state the key properties of the Schwarzian derivative that we will use. First, $S f$ is a holomoprhic quadratic differential on $X$. The quotient of the absolute value of a holomorphic quadratic differential and a metric is a function. Using the unique hyperbolic metric on $X$ we can take the sup-norm of this function to a define the sup-norm, $\|S f\|_{\infty}$, of the Schwarzian. This determines a metric on $P(X)$ by setting $d\left(\Sigma, \Sigma^{\prime}\right)=\|S f\|_{\infty}$. Furthermore, given any holomorphic quadratic differential $\Phi$ on $X$ there is a projective structure $\Sigma^{\prime}$ such that for the conformal map $f: \Sigma \longrightarrow \Sigma^{\prime}, S f=\Phi$. Therefore $P(X)$ is isomorphic to the vector space $Q(X)$ of holomorphic quadratic differentials on $X$.

A projective structure is Fuchsian if it is the quotient of a round disk in $\widehat{\mathbb{C}}$. There is a unique Fuchsian projective structure, $\Sigma_{F}$, in each $P(X)$. We will often be interested in the distance between an arbitrary projective structure $\Sigma \in P(X)$ and this unique Fuchsian projective structure. We therefore let $\|\Sigma\|_{F}=d\left(\Sigma, \Sigma_{F}\right)$.

As with any $(G, X)$-structure, a projective structure $\Sigma$ on $S$ determines a developing map

$$
D: \tilde{S} \longrightarrow \widehat{\mathbb{C}}
$$

and a holonomy representation

$$
\rho: \pi_{1}(S) \longrightarrow P S L_{2} \mathbb{C}
$$

satisfying (2.4). Now let $\Sigma_{t}$ be a smooth path of projective structures in $P(X)$. Then there is a smooth path of developing maps $D_{t}$ which determine vector fields $v_{t}$ on $\tilde{S}$. 
The developing maps, $D_{t}$, can be chosen to be conformal maps from $\tilde{X}$ to $\widehat{\mathbb{C}}$ which will make the vectors fields $v_{t}$ conformal on $\tilde{X}$.

Let $v(z)$ be a conformal vector field on a domain in $\widehat{\mathbb{C}}$. Then $v(z)=f(z) \frac{\partial}{\partial z}$ where $f$ is a holomorphic function. A conformal vector field is projective if its flow consists of elements of $P S L_{2} \mathbb{C}$. The space of projective fields is the Lie algebra $s l_{2} \mathbb{C}$ and $v(z)$ will be projective if and only if $f(z)$ is a quadratic polynomial. At each point $z$ in the domain let $s(z)$ be the unique projective vector field that best approximates $v$ at $z$. Note that $s(z)$ is obtained by taking the first three terms of the Taylor series of $f$ at $z$. Differentiating $s(z)$ we obtain an $s l_{2} \mathbb{C}$-valued 1 -form which can be canonically associated with a holomorphic quadratic differential. This quadratic differential is the Schwarzian derivative, $S v$, of the vector field $v$.

We now return to our path of projective structures $\Sigma_{t}$ in $P(X)$. The Schwarzian derivative of the conformal vector fields $v_{t}$ will be equivariant and therefore $S v_{t}$ will be a holomorphic quadratic differential on $X$. The norm $\left\|S v_{t}\right\|_{\infty}$ is the infinitesimal version of the metric on $P(X)$ and if we can bound it for all $t$ we bound the distance between $\Sigma_{0}$ and $\Sigma_{1}$.

We need one final fact about projective structures. The holonomy representation defines a map from $P(S)$ to the space $R(S)$ of representations of $\pi_{1}(S)$ in $P S L_{2} \mathbb{C}$ modulo conjugacy. We the have the following theorem.

Theorem 2.3 ([Hej75, Ea80, Hub80]). The holonomy map

$$
\text { hol : } P(S) \longrightarrow R(S)
$$

is a holomorphic, local homeomorphism.

\section{Hyperbolic cone-manifolds}

\subsection{Geometrically finite hyperbolic cone-manifolds}

Let $N$ be a compact manifold with boundary, $\mathscr{C}$ a collection of simple closed curves in the interior of $N$ and $M$ the interior of $N \backslash \mathscr{C}$. Let $g$ be a complete metric on the interior of $N$ that is a smooth Riemannian metric on $M$. We say that $g$ is a hyperbolic cone-metric if the following holds: First $g$ is a hyperbolic metric on $M$. Second, for points on $\mathscr{C}$ the metric has the form

$$
d r^{2}+\sinh ^{2} r d \theta^{2}+\cosh ^{2} r d z^{2}
$$

where $\theta$ is measured modulo some cone-angle $\alpha$. Note that the cone-angle must be locally constant on $\mathscr{C}$. Therefore there is a cone-angle associated to each component of $\mathscr{C}$.

Since the metric $g$ is complete the boundary $\partial N$ consists of tori and higher genus surfaces. Let $\partial_{0} N$ denote the higher genus components of the boundary. To develop a 
good deformation theory we need to assume that there metric $g$ has certain asymptotic behavior as we approach $\partial_{0} N$. We say that a hyperbolic, cone-metric $g$ is geometrically finite if the hyperbolic structure extends to a projective structure on $\partial_{0} N$. More explicitly $g$ is geometrically finite if for each $p \in \partial_{0} N$ there exists an open neighborhood of $p$ in $N$ and a map $\psi: V \longrightarrow \mathbb{H}^{3} \cup \widehat{\mathbb{C}}$ that is a homeomorphism onto its image and is an isometry on $V \cap \operatorname{int} M$. The restriction of $\psi$ to $V \cap \partial_{0} N$ will determine an atlas of charts to $\widehat{\mathbb{C}}$. Since hyperbolic isometries of $\mathbb{H}^{3}$ extend to projective transformations of $\widehat{\mathbb{C}}$ this atlas will determine a projective structure on $\partial_{0} N$.

Let $G F(N, \mathscr{C})$ be equivalence classes of geometrically finite hyperbolic conemanifolds on the pair $(N, \mathscr{C})$. If $g$ is a hyperbolic cone-metric on $(N, \mathscr{C})$ we refer to the induced projective structure on $\partial_{0} N$ as the projective boundary. The projective structure induces a conformal structure on $\partial_{0} N$. This is the conformal boundary.

Note that the round circles in the projective boundary are the boundary at infinity of hyperbolic planes in the hyperbolic manifold. As the 3-dimensional hyperbolic metric deforms these planes will not stay totally geodesic. This will be detected by the change in the projective boundary.

\subsection{Deformations of hyperbolic cone-manifolds}

A meridian for the pair $(N, \mathscr{C})$ is a simple closed curve $\gamma \subset \operatorname{int} N$ that bounds a disk in $N$ which intersects $\mathscr{C}$ in a single point. Each component of $\mathscr{C}$ has a unique meridian up to homotopy in $M=\operatorname{int} N \backslash \mathscr{C}$. Furthermore if $\rho$ is the holonomy of a cone-manifold structure on $(N, \mathscr{C})$ then $\rho(\gamma)$ will be elliptic (or the identity if the cone angle is a multiple of $2 \pi$ ) for all meridians $\gamma$.

On the other hand there certainly will be representations where not all meridians are elliptic. For this reason we let $R_{e}(M)$ be the subset of $R(M)$ where the meridians are elliptic or the identity. We then have the following theorem which is essentialy due to Thurston ([Th79]).

Theorem 3.1. The holonomy map

$$
\text { hol : } G F(N, \mathscr{C}) \longrightarrow R_{e}(M)
$$

is a local homeomorphism.

With this theorem our next goal is to give a local parameterization of $R(M)$. To do this we first need to define parameters. This local parameterization will be of a neighborhood in $R(M)$, not just a neighborhood in $R_{e}(M)$. These more general representations also have geometric signifigance. They correspond to Thurston's generalized Dehn surgery singularities. We will not explain the geometry of these singularities here.

Let

$$
\mathscr{L}_{\mathscr{M}}: R(M) \longrightarrow \mathbb{C}^{k}
$$


be the holomorphic map which assigns to each representation the $k$-tuple of complex lengths of the $k$-meridians of $(N, \mathscr{C})$. This is our first set of parameters.

The second set of parameters comes from the conformal boundary. Given a component $S$ of $\partial_{0} N$ we can define a map from $G F(N, \mathscr{C})$ to the Teichmüller space $T(S)$. This map assigns to each geometrically finite cone-manifold the conformal boundary structure on $S$. If $\rho \in R(M)$ is the holonomy of a cone-manifold in $G F(N, \mathscr{C})$ then by pre-composing this map with $\mathrm{hol}^{-1}$, we obtain a map $\partial_{S}$ from a neighborhood of $\rho$ in $R_{e}(M)$ to $T(S)$. Here we choose the unique branch of hol ${ }^{-1}$ that takes $\rho$ to the given geometrically finite cone-manifold. There is then a unique holomorphic extension of $\partial_{S}$ to a neighborhood of $\rho$ in $R(M)$.

Repeating the construction for each component of $\partial_{0} N$ and combining the maps we have a single map

$$
\partial: R(M) \longrightarrow T\left(\partial_{0} N\right)
$$

Strictly speaking $\partial$ is only defined for a neighborhood of $\rho$ in $R(M)$. We also note that there are examples of distinct geometrically finite hyperbolic cone-manifolds with the same holonomy representation. When this happens each manifold will define a different boundary map $\partial$.

Now we combine our two parameters. Define

$$
\Phi: R(M) \longrightarrow \mathbb{C}^{k} \times T\left(\partial_{0} N\right)
$$

by $\Phi(\rho)=\left(\mathscr{L}_{\mathscr{M}}(\rho), \partial(\rho)\right)$.

Theorem 3.2 ([HK98, HK, Br2]). Let $\rho$ be the holonomy of a geometrically finite cone-manifold. If the cone-angle is $\leq 2 \pi$ or the tube radius of the singular locus is $\geq \sinh ^{-1} 1 / \sqrt{2}$ then the map $\Phi$ is a holomorphic local homeomorphism.

Skecth of proof of theorem 3.2. By a theorem of Thurston

$$
\operatorname{dim}_{\mathbb{C}} R(M) \geq k+\operatorname{dim}_{\mathbb{C}} T\left(\partial_{0} N\right) .
$$

Since the map $\Phi$ is holomorphic if we can show that the derivative, $\Phi_{*}$, is injective at $\rho$ then $\Phi$ will be a local homeomorphism at $\rho$.

The first step in proving this injectivity is a Hodge theorem: Any tangent vector of $R(M)$ at $\rho$ that is in the kernel of $\partial_{*}$ is represented by a harmonic strain field $\eta$ on $\left(M, g_{\alpha}\right)$. Note there are some subtle issues to proving this Hodge theorem since our manifold is not compact and the metric is not complete. In particular, the harmonic strain field $\eta$ is only unique after making some choice of boundary conditions for the solution.

Next we would like to calculate the $L^{2}$-norm of $\eta$ on $M$. Theorem 2.1 tells how to calculate the $L^{2}$-norm of a harmonic strain field on a compact manifold with boundary. We can obtain a similar formula for harmonic strain fields on a geometrically finite manifold if the strain field fixes the conformal boundary. Analytically this is 
equivalent to $\partial_{*} \eta=0$ where $\partial_{*}$ is the tangent map of the boundary map $\partial$ from $R(M)$ to $T\left(\partial_{0} N\right)$. The pointwise norm of such conformal deformations will decay exponentially and the boundary term in (2.3) will limit to zero for surfaces exiting the geometrically finite end. This allows us to calculate the $L^{2}$-norm of $\eta$ even on the non-compact geometrically finite ends. In particular, we have

$$
\int_{M \backslash U}\|\eta\|^{2}+\|\nabla \eta\|^{2}=\int_{\partial U} * \nabla \eta \wedge \eta
$$

where $U$ is tubular neighborhood of the singular locus, even though $M \backslash U$ is not compact. Note that in general the $L^{2}$-norm will be infinite on all of $M$.

The final step is to calculate the boundary term. This is done in the following way. In a tubular neighborhood of the singular locus we can decompose $\eta$ as the sum of two strain fields, $\eta=\eta_{0}+\eta_{c}$. The first term, $\eta_{0}$, is an explicit model deformation completely determined by the derivatives of the complex lengths of the components of the singular locus and the meridians. The second term, $\eta_{c}$, is a correction term. It does not affect the complex length of the singular locus or the meridians. In particular, there is a vector field $v$ on a tubular neighborhood of the singular locus such that $\eta_{c}=\operatorname{sym} \nabla v$.

The advantage of this decomposition is that we can now decompose the boundary term:

$$
\int_{\partial U} * \nabla \eta \wedge \eta=\int_{\partial U} * \nabla \eta_{0} \wedge \eta_{0}+\int_{\partial U} * \nabla \eta_{c} \wedge \eta_{c} .
$$

The first term on the right can be calculated explicitly and will be non-positive if $\left(\mathscr{L}_{\mathscr{M}}\right)_{*} \eta=0$. The hard work is to show that the second term will always be nonpositive. Together this implies if $\Phi_{*} \eta=0$ then $\eta \equiv 0$ and therefore $\Phi_{*}$ is injective.

The following is a simple corollary of Theorem 3.1 and 3.2.

Corollary 3.3. Let $M_{\alpha}$ be a geometrically finite cone-manifold with cone angle $\alpha$ whose singular locus has a tubular neighborhood of radius $\geq \sinh ^{-1} \sqrt{2}$. Then, for $t$ near $\alpha$, there exists a one-parameter family of cone-manifolds $M_{t}$ with cone-angle $t$ and conformal boundary fixed.

We now set some notation that will be used throughout the rest of the paper. For any essential simple closed curve $\gamma$ in $M, L_{\gamma}(t)$ is the the length of $\gamma$ in $M_{t}$ and $\mathscr{L}_{\gamma}(t)$ is the complex length of $\gamma$. The imaginary part of $\mathscr{L}_{\gamma}(t)$ is denoted $\Theta_{\gamma}(t)$. For the special case of the singular locus, $L_{\mathscr{C}}(t)$ is the the sum of the lengths of all the components of the singular locus. Let $U_{t}(R)$ be the union of the $R$-tubular neighborhoods of the components of the singular locus. The $n$ components of the conformal boundary are denoted $X^{1}, \ldots, X^{n}$. The corresponding components of the projective boundary of $M_{t}$ are denoted $\Sigma_{t}^{1}, \ldots, \Sigma_{t}^{n}$.

The next theorem is key in controlling the geometry of the one-parameter family of cone-manifolds $M_{t}$. 
Theorem 3.4. The one parameter family of cone-manifolds $M_{t}$ can be realized by metrics $g_{t}$ with derivatives $\eta_{t}$ such that $\eta_{t}$ is a harmonic strain field outside of a radius 1 tube of the singular locus and

$$
\int_{M_{t} \backslash U_{t}(R)}\left\|\eta_{t}\right\|^{2}+\left\|\nabla_{t} \eta_{t}\right\|^{2} \leq \frac{2 L_{\mathscr{C}}(t)}{t^{2} \sinh ^{2} R}
$$

for all $R \geq 1$.

Skecth of proof of theorem 3.4. The proof has two parts: The construction of the metrics $g_{t}$ and the estimate on the $L^{2}$-norm of the strain field $\eta_{t}$. We will skip the first part and focus on the second.

The bound on the $L^{2}$-norm of $\eta_{t}$ follows the same pattern as the completion of the proof of Theorem 3.2. For each $t$ we decompose $\eta_{t}$ in $U_{t}$ as $\eta_{t}=\eta_{0}+\eta_{c}$ where $\eta_{0}$ is a model deformation and $\eta_{c}$ is a correction term. We have the same decomposition of the boundary term as in (3.1) and once again the correction term makes a non-positive contribution. The one difference we have is that the cone angle is now decreasing and so the term coming from the model deformation will be positive. However, we can make an explicit calculation to bound this positve number and see that

$$
\int_{\partial U_{t}(R)} * \nabla_{t} \eta_{0} \wedge \eta_{0} \leq \frac{2 L_{\mathscr{C}}(t)}{t^{2} \sinh ^{2} R}
$$

which gives the theorem.

We remark that the only significance of the tube radius 1 in the above theorem is that $1>\sinh ^{-1} 1 / \sqrt{2}$.

\section{The drilling theorems}

We call the process of decreasing the cone angle "drilling". In the three drilling theorems that follow we control various geometric quantites as we drill. Note that these drilling theorems only apply where the one-parameter family of cone-manifolds $M_{t}$ is defined. To be useful we need to know that we can drill the cone-angle a definite amount, say from $4 \pi$ to $2 \pi$ or $2 \pi$ to 0 . As we will see one consequence of the drilling theorems is that under certain conditions we can drill this definite amount.

In the first drilling theorem we estimate how the lengths of geodesics change as we drill.

Theorem 4.1 ([Br04]). For each $L>0$ there exists an $\varepsilon>0$ and an $A>0$ such that if $\gamma$ is a simple closed curve in $M$ with $L_{\gamma}(\alpha) \leq L$ and $L_{\mathscr{C}}(\alpha) \leq \varepsilon$ then

$$
e^{-A L_{\mathscr{C}}(\alpha)} L_{\gamma}(\alpha) \leq L_{\gamma}(t) \leq e^{A L_{\mathscr{C}}(\alpha)} L_{\gamma}(\alpha)
$$

and

$$
\left(1-A L_{\mathscr{C}}(\alpha)\right) \Theta_{\gamma}(\alpha) \leq \Theta_{\gamma}(t) \leq\left(1+A L_{\mathscr{C}}(\alpha)\right) \Theta_{\gamma}(\alpha)
$$

for all $t \leq \alpha$. 
Skecth of proof of theorem 4.1. To prove the first statement we need to bound the derivative $L_{\gamma}^{\prime}(t)$. There are two cases. The first case is when the length of $\gamma$ is bounded but not very short. In this case $\gamma$ will be in the thick part of $M_{t}$. We then use the $L^{2}$-bounds given by Theorem 3.4 along with Theorem 2.2 to find a pointwise bound on $\eta_{t}$ for all points on $\gamma$. This, in turn, bounds the derivative.

The second case is when $\gamma$ is very short. By a version of the Margulis Lemma, $\gamma$ will have a large tubular neighborhood $U$. We decompose $\eta_{t}$ on $U$ into a model term, $\eta_{0}$, and a correction term, $\eta_{c}$, as before. A bound on the $L^{2}$-norm of the model will bound the derivative $L_{\gamma}^{\prime}(t)$. The model term, $\eta_{0}$, is like a deformation of a component of the singular locus that does not change the cone angle. As in the proof of Theorem 3.2 this determines the sign of the boundary term. However, in this case the sign will be positive since the torus $\partial U$ has the opposite orientation of the boundary torus in Theorem 3.2. This is because we are calculating the $L^{2}$-norm on $U$ rather than its complement. The sign of the boundary term for $\eta_{c}$ will also be positive for the same reason. This last fact, together with Theorem 3.4 gives the desired bound on the $L^{2}$-norm of $\eta_{0}$ on $U$.

The second statement of the theorem is proved by a similar method.

In the next drilling theorem we bound the change in the projective boundary of $M_{t}$ as we drill. This should be thought of as controlling the geometry of the geometrically finite ends.

Theorem 4.2 ([Br04]). There exists a $C$ depending only on $\alpha$, the injectivity radius of the unique hyperbolic metric on $X^{i}$ and $\left\|\Sigma_{\alpha}^{i}\right\|_{F}$ such that

$$
d\left(\Sigma_{\alpha}^{i}, \Sigma_{t}^{i}\right) \leq C L_{\mathscr{C}}(\alpha)
$$

for all $t \leq \alpha$.

Skecth of proof of theorem 4.2. The derivative of the path $\Sigma_{t}^{i}$ in $P(X)$ is a path of quadratic differentials $\Phi_{t}^{i}$ in $Q\left(X^{i}\right)$. We will bound the size of $\Phi_{t}^{i}$.

A embedded round disk $D$ in $\Sigma_{t}^{i}$ bounds an embedded half space $H$ in $M_{t}$. The first step is to show that a bound on the $L^{2}$-norm of $\eta_{t}$ on $H$ implies a bound on the sup norm of $\Phi_{t}^{i}$ with respect to the hyperbolic metric on $D$. The proof of this fact follows our previous theme. We decompose the harmonic strain field $\eta_{t}$ into a model term, $\eta_{0}$, completely determined by $\Phi_{t}^{i}$ and a correction term $\eta_{c}$. Once again the $L^{2}$-norm of $\eta_{t}$ on $H$ is the sum of the $L^{2}$-norms of $\eta_{0}$ and $\eta_{c}$ so Theorem 3.4 bounds the $L^{2}$-norm of $\eta_{0}$. Since $\eta_{0}$ is explicitly determined by $\Phi_{t}^{i}$ this bounds the sup norm of $\Phi_{t}^{i}$.

Notice that we have only bounded the sup norm with respect to the hyperbolic metric on $D$, not with respect to the hyperbolic metric on $X^{i}$. To finish the proof we need to compare the two metrics. In particular for every point $z$ we can find a disk $D$ containing $z$ where the ratio of the two metrics is bounded by constants depending only on the injectivity radius of $X^{i}$ and $\left\|\Sigma_{t}^{i}\right\|_{F}$.

Together, the previous two results give enough control to prevent any degeneration as the cone angle decreases. In particular we have the following theorem: 
Theorem 4.3 ([Br04]). For any $\alpha>0$ there exists an $\ell>0$ such that if $M_{\alpha}$ is a geometrically finite cone-manifold with $L_{\mathscr{C}}(\alpha) \leq \ell$ and tube radius $>\sinh ^{-1} 1 / \sqrt{2}$ then the one parameter family is defined for all $t \in[0, \alpha]$.

The final drilling theorem is also the strongest. Theorem 1.1 is a special case. It essentially implies the previous two drilling theorems although the dependence of the constants on the length of the singular locus is not so clear.

Theorem 4.4 ([BB04]). For any $K>1$ there exists an $\ell>0$ depending only on $K$ and $\alpha$ such that the following holds. If $L_{\mathscr{C}}(\alpha) \leq \ell$ there is for each $t \in[0, \alpha]$ a standard neighborhood $\mathbb{T}_{t}(\mathscr{C})$ of the singular locus $\mathscr{C}$ and a $K$-bi-Lipschitz diffeomorphism of pairs

$$
h_{t}:\left(M_{\alpha} \backslash \mathbb{T}_{\alpha}(\mathscr{C}), \partial \mathbb{T}_{\alpha}(\mathscr{C})\right) \longrightarrow\left(M_{t}, \mathbb{T}_{t}(\mathscr{C}), \partial \mathbb{T}_{t}(\mathscr{C})\right)
$$

Skecth of proof of theorem 4.4. Recall that in Theorem 3.4 we constructed a family of metrics, $M_{t}=\left(M, g_{t}\right)$, whose derivative was the harmonic strain fields $\eta_{t}$. For points in the thick part of $M_{t}$ the combination of Theorems 3.4 and 2.2 bound the pointwise norm of $\eta_{t}$. Therefore, on the thick part of $M_{\alpha}$ the identity map on $M$ is a $K$-biLipschitz map from $\left(M, g_{\alpha}\right)$ to $\left(M, g_{t}\right)$ when the singular locus is sufficiently short.

We are left to extend $h_{t}$ to the thin parts of $M_{\alpha}$ which will be a collection of Margulis tubes. This is done by hand. The maps $h_{t}$ are $K$-bi-Lipschitz on the boundary of these Margulis tubes and we build an explicit extension of this map inside the tube. The construction is somewhat tedious and we will not describe it here.

\section{Geometric inflexibility}

A nice application of the boundary formula of Theorem 2.1 is to show exponential decay of the $L^{2}$-norm. Essentially, the formula shows that the $L^{2}$-norm of a harmonic strain field on the 3-manifold is equal to its $L^{2}$-norm on the boundary. A function whose integral equals its boundary values will be exponential. This leads to the exponential decay of harmonic strain fields. Here is the precise theorem:

Theorem 5.1 ([BB]]). Let $M$ be a complete hyperbolic 3-manifold with boundary and $\eta$ a harmonic strain field on $M$ that has finite $L^{2}$-norm. Let $M(t)$ be the subset of $M$ consisting of the points that are distance $t$ or greater from $\partial M$. Then

$$
\int_{M(t)}\|\eta\|^{2}+\|\nabla \eta\|^{2} \leq e^{-2 t} \int_{M}\|\eta\|^{2}+\|\nabla \eta\|^{2}
$$

Skecth of proof of theorem 5.1. The first step is to see that Theorem 2.1 applies to $M$ and $M(t)$ to get

$$
\int_{M(t)}\|\eta\|^{2}+\|\nabla \eta\|^{2}=\int_{\partial M(t)} * \nabla \eta \wedge \eta
$$

even thought $M(t)$ is not compact. The second fact we need is the following inequality

$$
\|\eta\|^{2}+\|\nabla \eta\|^{2} \geq 2\|* \nabla \eta \wedge \eta\|
$$


Now let

$$
f(t)=\int_{M(t)}\|\eta\|^{2}+\|\nabla \eta\|^{2}
$$

which we can rewrite as

$$
f(T)=\int_{T}^{\infty} \int_{\partial M(t)}\left(\|\eta\|^{2}+\|\nabla \eta\|^{2}\right) d A d t
$$

where $d A$ is the area form on $\partial M(t)$. Differentiating we have

$$
\begin{aligned}
-f^{\prime}(t) & =\int_{\partial M(t)}\left(\|\eta\|^{2}+\|\nabla \eta\|^{2}\right) d A \\
& \geq 2 \int_{\partial M(t)} * \nabla \eta \wedge \eta \\
& \geq 2 f(t) .
\end{aligned}
$$

Integrating both sides of the final inequality gives the theorem.

McMullen has proven a similar theorem, using entirely different methods, for harmonic strain fields arising from quasi-conformal deformations of complete hyperbolic 3-manifolds. He calls his theorem "geometric inflexibilty" which we follow.

One application of the geometric inflexibility theorem is stronger versions of the drilling theorems. For example, the bounds on the change in length of a closed geodesic given by Theorem 4.1 will decay exponentially in the distance of the geodesic from the singular locus.

To apply geometric inflexibility to Theorem 4.2 we need another definition. A geometrically finite cone-manifold will have a convex core which will be a submanifold with boundary consisting of convex surfaces. There will be one component of the boundary the convex core facing each component of the projective boundary. For $\gamma$ a closed geodesic and $\Sigma$ a component of the projective boundary let $d(\gamma, \Sigma)$ be the shortest distance from $\gamma$ to the component of the boundary of the convex core facing $\Sigma$.

Theorem 5.2. There exists $C_{1}$ and $C_{2}$ depending only on $\alpha$, the injectivity radius of the unique hyperbolic metric $X^{i}$ and $\left\|\Sigma_{\alpha}^{i}\right\|_{\infty}$ such that

$$
d\left(\Sigma_{\alpha}^{i}, \Sigma_{t}^{i}\right) \leq C_{1} e^{-C_{2} d\left(\mathscr{C}, \Sigma_{\alpha}^{i}\right)} L_{\mathscr{C}}(\alpha)
$$

for all $t \leq \alpha$

\section{Applications to the Bers' slice}

A Kleinian group is a discrete subgroup of $P S L_{2} \mathbb{C}$. Here we will restrict to the special class of Kleinian groups that arise as the image of holonomy representations of 
projective structures on a closed surface $S$. The advantage of restricting to this class is that we can use the topology and metric on the space of projective structures to study the family of Kleinian groups.

For a more precise definition let $U(X)$ be the set of projective structures in $P(X)$ whose developing map is injective. For every projective $\Sigma \in U(X)$ the image of the holonomy representation $\rho\left(\pi_{1}(S)\right)$ will act properly discontinuously on the image of the developing map, $D(\tilde{S})$. Since the developing map is injective, $D(\tilde{S})$ will be an open topological disk in $\widehat{\mathbb{C}}$. A group that acts properly discontinuously on a open subset of $\widehat{\mathbb{C}}$ will be discrete and therefore $\rho\left(\pi_{1}(S)\right)$ is a Kleinian group.

A Kleinian group is quasifuchsian if it acts properly discontinuously on two disjoint open disks in $\widehat{\mathbb{C}}$. Let $T(X)$ be the subset of $U(X)$ where the image of the holonomy representation is quasifuchsian. The space $T(X)$ is a Bers' slice of the space of all quasifuchsian groups. Let $\rho$ be the holonomy of a projective structure in $T(X)$ and let $\Omega$ be the open disk, disjoint from $D(\tilde{S})$, on which $\rho\left(\pi_{1}(S)\right)$ acts properly discontinuously. Then $\Omega / \rho\left(\pi_{1}(S)\right)$ defines a projective structure and hence a conformal structure on $\bar{S}$, where $\bar{S}$ is the oriented surface $S$ with the orientation reversed. This defines a map $T(X) \longrightarrow T(\bar{S})$ which we call the Bers' isomorphism for reasons that the following Theorem make apparent.

Theorem 6.1 ([Bers60]). The map $T(X) \longrightarrow T(\bar{S})$ is a homeomorphism.

The Bers' slice, $T(X)$, is the simplest example of a quasi-conformal deformation space. The above theorem implies that $T(X)$ is canonically identified with Teichmüller space. Since $U(X)$ is bounded in $P(X)$ the closure $\overline{T(X)}$ is a compactification of Teichmüller space.

In what follows we will continually refer to various objects determined by a projective structure $\Sigma$ in $U(X)$. First there is the holonomy representation $\rho$. Since its image is a Kleinian group isomorphic to $\pi_{1}(S)$ the quotient $\mathbb{H}^{3} / \rho\left(\pi_{1}(S)\right)$ is a hyperbolic 3-manifold $M$ homotopy equivalent to $S$. By the previous theorem if $\Sigma$ is in $T(X)$ it will also determine a conformal structure $Y$ in $T(\bar{S})$. If we are examining sequences of projective structures we will add indices and decorations to $\Sigma$. These will be promoted to all the corresponding objects.

We now state three conjectures about these spaces. Note that all of these conjectures have versions that apply to more general families of Kleinian groups.

The first two conjecture are from Bers' seminal paper [Bers70] which began the study of the the space $U(X)$.

The following conjecture is usually called the Bers' density conjecture:

Conjecture 6.2 ([Bers70]). $U(X)=\overline{T(X)}$

A projective structure in $\partial T(X)=\overline{T(X)} \backslash T(X)$ is a cusp if the image of the holonomy representation contains cusps. 


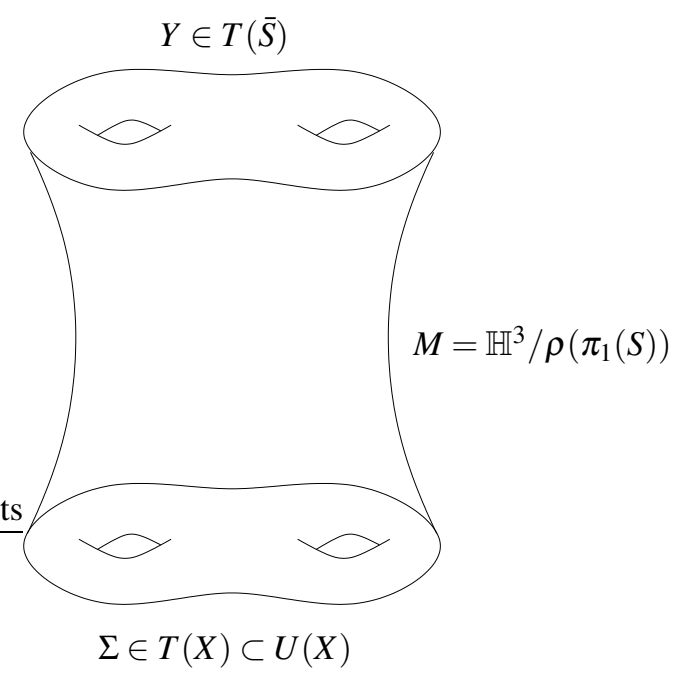

Figure 1: A quasifuchian manifold

Conjecture 6.3 ([Bers70]). Cusps are dense in the boundary of $T(X)$.

The final conjecture we will state is Thurston's ending lamination conjecture. To do so we need to define an ending lamination. We will put off doing this till later and at this point simply state that to each $\Sigma \in U(X)$ we can define an end invariant which is determined by the hyperbolic manifold $M$.

Conjecture 6.4. An element of $U(X)$ is uniquely determined by its end-invariant.

We note that all three of these conjecture are now known to be true. In fact the ending lamination conjecture implies the previous two conjectures. Our purpose here is to describe how the deformation theory developed in this paper can be used to approach these conjectures. At present this approach still has significant gaps (at least for the first and third conjecture) but if completed it would provide new proofs of all three conjectures.

\subsection{The Bers' density conjecture}

In its most general form, the density conjecture states that every finitely generated Kleinian group is a limit of geometrically finite Kleinian groups. Very recently this complete version of the conjecture has been proven. To do so one needs to combine a number of results: the ending lamination conjecture ([Min02, BCM04]), tameness ([Bon86], [Ag04], [CG04]) and various theorems on limits of Kleinian groups ([Th, Osh90, Brk00, KS02]). Although we will only address a very special case of the density conjecture here, the methods described apply in greater generality (see [BB04]). 
Conjecture 6.2 was the original version of the density conjecture. In [Br02] we proved the following result:

Theorem 6.5. Let $\Sigma$ be a projective structure in $U(X)$ such that the image of the holonomy has no parabolics. Then $\Sigma \in \overline{T(X)}$.

The theorem is proved in two cases. Let $M=\mathbb{H}^{3} / \rho\left(\pi_{1}(S)\right)$ be the quotient hyperbolic 3-manifold. Then $M$ has bounded geometry if there is a lower bound on the length of any closed geodesic in $M$. Otherwise $M$ has unbounded geometry. Minsky proved Theorem 6.5 when $M$ has bounded geometry. Our contribution was the case when $M$ has unbounded geometry. We will give a brief sketch of the proof, emphasizing those parts that use the deformation theory we have described in this paper.

The starting point is the following tameness theorem of Bonahon:

Theorem 6.6 ([Bon86]). The manifold $M$ is homeomorphic to $S \times(0,1)$.

A simple closed curve $\gamma$ in $S \times(0,1)$ is unknotted if it is isotopic to a simple closed curve on $S \times\{1 / 2\}$.

Theorem 6.7 ([Br02]). Let $\gamma$ be an unknotted, simple closed geodesic in $M$ and assume that the product structure is chosen such that $\gamma$ lies on $S \times\{1 / 2\}$. Then there is a geometrically finite hyperbolic cone-manifold $M_{\gamma}$ with the following properties:

(i) The singular locus has a single component with cone angle $4 \pi$.

(ii) The length of the singular locus in $M_{\gamma}$ is equal to the length of $\gamma$ in $M$. The tube radius of the singular locus in $M_{\gamma}$ is greater than or equal to the tube radius of $\gamma$ in $M$.

(iii) $M$ and $M_{\gamma}$ are isometric on $S \times(0,1 / 2)$.

The construction of $M_{\gamma}$ is similar to the construction of grafting of complex projective structures. Although we will not go through it here, it is not difficult. The proof that $M_{\gamma}$ is geometrically finite is more involved. For a proof in the above case see [Br02]. A proof in a more general setting can be found in [BB04]. An expository account can be found in [BB03].

To apply Theorem 6.7 we use the following theorem of Otal:

Theorem 6.8 ([Ot95, Ot03]). There exists an $\varepsilon_{\text {unknot }}>0$ depending only on the genus of $S$ such that if $\gamma$ is a closed geodesic in $M$ of length less than $\varepsilon$ then $\gamma$ is unknotted.

Now assume that $M$ has unbounded geometry. Then there exists a sequence of closed geodesics $\gamma_{i}$ whose lengths limits to zero. In particular we can assume that $L_{\gamma_{i}}(M) \leq \min \left\{\varepsilon_{\text {unknot }}, \ell\right\}$ for all $\gamma_{i}$ where $\ell$ is the constant in Theorem 4.3. Then for each $\gamma_{i}$, Theorem 6.7 gives us a cone-manifold $M_{i}$ with cone-angle $4 \pi$. Furthermore, 
by (3) of Theorem 6.7 the component of the projective boundary on $S \times\{0\}$ of $M_{i}$ will be the original projective structure $\Sigma$.

Next we apply Theorem 4.3 to decrease the cone-angle to $2 \pi$ obtaining a quasifuschsian manifold $M_{i}^{\prime}$. Let $\Sigma_{i}$ be the $S \times\{0\}$ component of the projective boundary of $M_{i}^{\prime}$. By Theorem 4.2 there exists a $C$ such that $d\left(\Sigma, \Sigma_{i}\right) \leq C L_{\gamma_{i}}\left(M_{i}\right)=C L_{\gamma_{i}}(M)$ and therefore $\Sigma_{i} \rightarrow \Sigma$, as desired. This completes the sketch of the proof of Theorem 6.5 in the case of unbounded geometry.

What if $M$ has bounded geometry? As we have already mentioned Minsky proved Theorem 6.5 in this case. He did so by proving the ending lamination conjecture for manifolds with bounded geometry. One might hope to find a direct approach to Conjecture 6.2 based on the methods outlined here.

There are two problems. First, there may not be a sequence of unknotted geodesics. Second, even if we are fortunate enough to have a sequence of unknotted geodesics the singular locus in the corresponding cone-manifolds will not be short and we won't be able to apply Theorem 4.3.

We can circumvent the first problem by lifting to a cover. The second problem is more serious. For a cone-manifold whose singular locus is not short to guarantee that the manifold can be deformed to cone angle zero we need to assume the the singular locus has a large tubular neighborhood. In particular we have the following theorem whose proof is beyond the scope of this paper:

Theorem 6.9. Given any $\alpha, L>0$ there exists an $R>0$ such that the following holds. Let $M_{\alpha}$ be a geometrically finite hyperbolic cone-manifold with cone angle $\alpha$ and $L_{\mathscr{C}}(\alpha) \leq L$ and assume that the singular locus has a tube radius $\geq R$. Then the oneparameter family $M_{t}$ exists for all $t \in[0, \alpha]$.

The next theorem allows us to circumvent both above problems by lifting to a cover. It is direct corollary of Theorems 2.1 and 4.3 of [FG01].

Theorem 6.10. Let $\gamma$ be a closed geodesic in a hyperbolic 3-manifold $M$ with $M$ homeophic $S \times(0,1)$. Given $R>0, M$ has a finite cover $\hat{M}$ for which $\gamma$ has a homeomorphic lift $\hat{\gamma}$ that is unknotted and has a tubular neighborhood of radius $>R$.

The tradeoff is that we are now working in a cover instead of with the original manifold. Because of this we can only prove that the projective structure lies in the boundary of the universal Teichmüller space. We now define this space. Let $P(1)$ be the space of bounded, holomorphic quadratic differentials on the unit disk $\Delta$ in $\widehat{\mathbb{C}}$. Given $\Phi \in P(1)$ there exists a locally conformal map $f: \Delta \longrightarrow \widehat{\mathbb{C}}$ with $S f=\Phi$. This $f$ is unique up to post-composition by elements of $P S L_{2} \mathbb{C}$. Let $U(1) \subset P(1)$ be those quadratic differentials where $f$ is injective (or univalent in the language of complex analysis) and let $T(1) \subset U(1)$ be those quadratic differentials where $f$ extends to a quasi-conformal homeomorphism of all of $\widehat{\mathbb{C}}$. The space $T(1)$ is usually called 
the universal Teichmüller space because all Teichmüller spaces $T(X)$ embed in $T(1)$. That is if $\Sigma$ is a projective structure in $P(X)$ the the universal cover, $\tilde{\Sigma}$, is a projective structure in $P(1)$ and the map taking $\Sigma$ to $\tilde{\Sigma}$ is an isometry.

Bers $^{2}$ made the following conjecture:

Conjecture 6.11. $U(1)=\overline{T(1)}$

This conjecture is known to be false. Counterexamples where found by Gehring ([Geh78]) and later Thurston ([Th86]). However, we can prove the following theorem

Theorem 6.12. Let $\Sigma \in U(X)$ be a projective structure whose holonomy does not have parabolics. Then $\tilde{\Sigma} \in \overline{T(1)}$.

Proof. In the course of proving Theorem 6.6, Bonahon shows that $M$ has a sequence of closed geodesics $\gamma_{i}$ with bounded length and $d\left(\gamma_{i}, \Sigma\right) \rightarrow \infty$. (Recall that $d\left(\gamma_{i}, \Sigma\right)$ is the distance from the component of the convex core boundary facing $\Sigma$ to $\gamma_{i}$.) Now, for each $\gamma_{i}$, apply Theorem 6.10 to obtain a cover to which we can apply both Theorem 6.7 and Theorem 6.9. That is, in the cover, $\gamma_{i}$ lifts to an unknotted geodesic $\hat{\gamma}_{i}$ along which we can graft to obtain a geometrically finite cone-manifold $\hat{M}_{i}$. The tube radius of singular locus will be sufficiently large so that we can decrease the cone angle to $2 \pi$ and obtain a quasifuchsian manifold $\hat{M}_{i}^{\prime}$.

Let $\hat{\Sigma}_{i}$ be the corresponding cover of the projective structure $\Sigma$. Then $\hat{\Sigma}_{i}$ is a component of the projective boundary of the cone-manifold $\hat{M}_{i}$. After the cone-manifold deformation this projective structure deforms to a projective structure $\hat{\Sigma}_{i}^{\prime}$. By Theorem 5.2 we have

$$
d\left(\hat{\Sigma}_{i}, \hat{\Sigma}_{i}^{\prime}\right) \leq e^{-k d\left(\hat{\Sigma}_{i}, \hat{\gamma}_{i}\right)} L_{\gamma_{i}}\left(\hat{M}_{i}\right) .
$$

Now $d\left(\hat{\Sigma}_{i}, \hat{\gamma}_{i}\right)=d(\Sigma, \gamma)$ which limits to zero and $L_{\hat{\gamma}_{i}}\left(\hat{M}_{i}\right)=L_{\gamma_{i}}(M)$ is bounded so the left hand side of (6.1) limits to zero. Therefore $\tilde{\Sigma}_{i} \rightarrow \tilde{\Sigma}=\tilde{\Sigma}_{i}$ in $U(1)$ as desired.

\subsection{Cusps are dense}

The conjugacy classes of parabolics in $\rho\left(\pi_{1}(S)\right)$ correspond to disjoint simple closed curves on $S$. In particular there are at most $3 g-3$ conjugacy classes. A cusp whose holonomy has this maximal number of conjugacy classes of parabolics is called a maximal cusp. McMullen proved the following strong version of Conjecture 6.3.

Theorem 6.13 ([Mc91]). Maximal cusps are dense on the boundary of $T(X)$.

Proof. Our proof will follow McMullen's except that we will replace his key estimate with Theorem 4.2. The part of the argument that we copy can be found on p. 221 of [Mc91].

\footnotetext{
${ }^{2}$ Conjectures 6.2 and 6.11 are labelled Conjectures II and I in [Bers70]. After stating Conjecture II Bers remarks "This would, of course, be a consequence of Conjecture I". This is not obvious to this author.
} 
The first step is to note that projective structures whose holonomy does not have parabolics are dense in $\partial T(X)$. Let $\Sigma$ be such a projective structure. To prove the theorem we need to show that $\Sigma$ is approximated by maximal cusps. To do this McMullen finds projective structures $\Sigma_{i} \in T(X)$ that limit to $\Sigma$ with the following property: The projective structures $\Sigma_{i}$ correspond to conformal structures $Y_{i} \in T(\bar{S})$. For each $Y_{i}$ there is a pants decompositions $P_{i}$ such that $L_{P_{i}}\left(Y_{i}\right) \rightarrow 0$ where the length is measured in the unique hyperbolic metric on $Y_{i}$.

On $Y_{i}$ we may assume that $L_{P_{i}}\left(Y_{i}\right) \leq \frac{1}{2} \min \left(\varepsilon_{\text {unknot }}, \ell\right)$ where $\ell$ is the constant in Theorem 4.3. By Bers' inequality ([Bers70]) this implies that $L_{P_{i}}\left(M_{i}\right) \leq \min \left(\varepsilon_{\text {unknot }}, \ell\right)$ where $M_{i}$ is the quasifuchsian hyperbolic manifold determined by $\Sigma_{i}$. Now view $M_{i}$ as a cone-manifold with singular locus $P_{i}$ and cone angle $2 \pi$. Since $L_{P_{i}}\left(M_{i}\right) \leq \ell$ we can decrease the cone angle of $M_{i}$ to zero to obtain a manifold $M_{i}^{\prime}$ with rank two cusps. The projective structure $\Sigma_{i}$ is a component of the projective boundary of $M_{i}$ and it deforms to a projective structure $\Sigma_{i}^{\prime}$. By Theorem 4.2

$$
d\left(\Sigma_{i}, \Sigma_{i}^{\prime}\right) \leq K L_{P_{i}}\left(M_{i}\right)
$$

and therefore

$$
\lim _{i \rightarrow \infty} \Sigma_{i}^{\prime}=\lim _{i \rightarrow \infty} \Sigma_{i}=\Sigma
$$

What remains to show is that the $\Sigma_{i}^{\prime}$ are maximal cusps. Let $\hat{M}_{i}$ be the cover of $M_{i}^{\prime}$ corresponding to the boundary component $\Sigma_{i}^{\prime}$. Since $L_{P_{i}}\left(M_{i}\right) \leq \varepsilon_{\text {unknot }}$, the geodesic representative of $P_{i}$ is unknotted in $M_{i}$ and therefore $M_{i}^{\prime}$ is homeomorphic to $S \times(0,1)$ with the curves $P_{i}$ removed from the halfway surface $S \times\{1 / 2\}$. Therefore $\hat{M}_{i}$ is homeomorphic to $S \times(0,1)$ and every curve in $P_{i}$ will be parabolic. This implies that $\Sigma_{i}^{\prime}$ is a maximal cusp.

There are versions of the density of cusps for more general quasiconformal deformation spaces in [CCHS03] and [CH04]. Both of these papers are generalizations of McMullen's methods. We note that our methods can also be used to prove these generalizations. See $\S 8$ of [Br04].

\subsection{The ending lamination conjecture}

The ending lamination conjecture is a classification of Kleinian groups isomorphic to a fixed group. The complete conjecture has recently be proven by Brock, Canary and Minsky ([Min02, BCM04]), completing a program of Minsky. In this section we will discuss an alternate approach to the conjecture. The approach is motivated by a theorem of R. Evans, which we will mention below. We also note, that this approach, if successful, uses some of Minsky's results in a key way and is heavily influenced by his ideas. The main difference is that we do not use the "model manifold".

The classifying objects are end-invariants which are objects associated to the surfaces that compactify the higher genus ends of the hyperbolic manifold. For groups 
without parabolics these invariants are either a conformal structure or a filling lamination on the surface compactifying the end. With parabolics the situation is more complicated. As usual, we will restrict to groups without parabolics.

For $\Sigma \in U(X)$ the corresponding manifolds $M$ has two ends both compactified by $S$. On $S \times\{0\}$ the end-invariant is always the conformal structure $X$. If $\Sigma \in T(X)$ then the end-invariant for $S \times\{1\}$ will also be a conformal structure. In this case the conformal structure will be the image of $\Sigma$ in $T(\bar{S})$ under the Bers' isomorphism. For $\Sigma \in U(X) \backslash T(X)$ the end-invariant is a lamination. To define it we recall that there is a sequence of closed geodesics, $\gamma_{i}$, whose length is bounded and such that $d\left(\gamma_{i}, \Sigma\right) \rightarrow 0$. Furthermore, Bonahon ([Bon86]) shows that these geodesics can be chosen to be homotopic to simple closed curves on $S \times\{1 / 2\}$. As simple closed curves on $S$, the $\gamma_{i}$ will limit to a lamination $\lambda$. Most importantly this ending lamination will not depend on the initial choice of geodesics. This is also a result of Bonahon ([Bon86]).

The following theorem of Minsky shows the importance of the ending lamination. It is a combination of his proof of the ending lamination conjecture for bounded geometry manifolds and one of the first steps in the proof of the general conjecture.

Theorem 6.14 ([Min01]). Let $\Sigma$ and $\Sigma^{\prime}$ be projective structures in $U(X)$ and assume that the corresponding hyperbolic manifolds $M$ and $M^{\prime}$ have the same end-invariant. Then either:

(i) $M$ and $M^{\prime}$ are isometric.

(ii) $M$ and $M^{\prime}$ both have unbounded geometry and there exists a sequence of simple closed curves $\gamma_{i}$ so that both $L_{\gamma_{i}}(M)$ and $L_{\gamma_{i}}\left(M^{\prime}\right)$ limit to zero.

We empasize that (1) and (2) are not mutually exclusive. In fact the goal is to show that (1) always holds. This is exactly the ending lamination conjecture.

For $\gamma$ a simple closed curve on $S$, let $U(X, \gamma) \subset U(X)$ be those projective structures in $U(X)$ where the conjugacy class of $\gamma$ is parabolic under the holonomy representation. Let $d_{\mathscr{C}}(\gamma, X)$ be the distance between $\gamma$ and $X$ in the curve complex. That is $d_{\mathscr{C}}(\gamma, X)$ is the minimum number $k$ such that there exist $k+1$ essential simple closed curves, $\beta_{0}, \ldots, \beta_{k}$ on $S$ with $\beta_{0}=\gamma, \beta_{i}$ and $\beta_{i+1}$ disjoint, and $\beta_{k}$ a bounded length curve in the hyperbolic metric on $X$.

While we believe the following conjecture is interesting in its own right, as we will see below it also implies the ending lamination conjecture for manifolds in $U(X)$ with unbounded geometry.

Conjecture 6.15. There exists a constants $C_{1}$ and $C_{2}$, depending only on the genus of $S$, such that the diameter of $U(X, \gamma)$ in $P(X)$ is bounded by $C_{1} e^{-C_{2} d_{\mathscr{C}}(X, \gamma)}$.

Our motivation for this conjecture is as follows. The distance $d_{\mathscr{C}}(\gamma, X)$ gives a 
lower bound on the thickness of the convex core of every manifold in $U(X, \gamma)$. One then wants to combine this with geometric inflexibility to obtain the desired bound.

We now show how Theorem 6.14 and Conjecture 6.15 together imply the ending lamination conjecture for projective structures in $U(X)$ with unbounded geometry and no cusps.

Let $\Sigma$ and $\Sigma^{\prime}$ be as in Theorem 6.14 and assume that $M$ and $M^{\prime}$ have unbounded geometry. Let $\gamma_{i}$ be the sequence given by (2) in Theorem 6.14. By Theorem 6.5 there exists a sequence $\Sigma_{i}$ in $T(X)$ converging to $\Sigma$. Let $M_{i}$ be the associated hyperbolic 3-manifolds. After passing to a subsequence we can assume that $L_{\gamma_{i}}\left(M_{i}\right) \rightarrow 0$. Now repeating the construction in the proof of Theorem 6.13 , for each $\Sigma_{i}$ we can find a cusp $\hat{\Sigma}_{i}$ in $U\left(X, \gamma_{i}\right)$ such that

$$
d\left(\Sigma_{i}, \hat{\Sigma}_{i}\right) \leq C L_{\gamma_{i}}\left(M_{i}\right)
$$

Therefore the sequence $\hat{\Sigma}_{i}$ converges to $\Sigma$. We similarly find a sequence $\hat{\Sigma}_{i}^{\prime}$ converging to $\Sigma^{\prime}$ with each $\hat{\Sigma}_{i}^{\prime}$ in $U\left(X, \gamma_{i}\right)$. Finally we note that the $\gamma_{i}$ converge to the ending lamination so $d_{\mathscr{C}}\left(\gamma_{i}, X\right)$ limits to infinity. Conjecture 6.15 then implies that both sequences $\hat{\Sigma}_{i}$ and $\hat{\Sigma}_{i}^{\prime}$ have the same limit so $\Sigma=\Sigma^{\prime}$.

Note that, in the above argument, if we replace the curves $\gamma_{i}$ with pants decompositions $P_{i}$ such that

$$
\lim _{i \rightarrow \infty} L_{P_{i}}(M)=\lim _{i \rightarrow \infty} L_{P_{i}}\left(M^{\prime}\right)=0
$$

then $\hat{\Sigma}_{i}$ and $\hat{\Sigma}_{i}^{\prime}$ will be maximal cusps. Since maximal cusps are uniquely determined by the pants decompositon $\hat{\Sigma}_{i}=\hat{\Sigma}_{i}^{\prime}$ and the corresponding limits, $\Sigma$ and $\Sigma^{\prime}$, are equal without appealing to Conjecture 6.15. This argument, due to Evans, leads to the following theorem that we mentioned at the begining of this section.

Theorem 6.16 ([Ev03]). Let $\Sigma$ and $\Sigma^{\prime}$ be projective structures in $U(X)$ with corresponding hyperbolic manifolds $M$ and $M^{\prime}$. Assume that there exist a sequence of pants decompositions $P_{i}$ with

$$
\lim _{i \rightarrow \infty} L_{P_{i}}(M)=\lim _{i \rightarrow \infty} L_{P_{i}}\left(M^{\prime}\right)=0
$$

Then $\Sigma=\Sigma^{\prime}$.

We remark that having such a shrinking pants decomposition is not as restrictive as it may seem. In fact, the density of maximal cusps (Theorem 6.13) implies that there is a dense $G_{\delta}$ of such manifolds in $\partial T(X)$. Furthermore, if $M$ has such a sequence of pants decompositions and $M^{\prime}$ has the same ending lamination as $M$ then the lengths of the same sequence of pants will limit to zero in $M^{\prime}$. This last statement is proven in [Min02] and is a large part of the proof of the ending lamination conjecture. Namely, in [Min02], Minsky constructs a model for $M$ that is completely determined by combinatorial information coming from the ending lamination. He then shows that there is a Lipschitz map from this model to the hyperbolic manifold $M$. Furthermore, 
every sufficiently short curve in $M$ will also be short in the model. Therefore, if $M^{\prime}$ has the same ending lamination as $M$ then it will have the same model and the same short geodesics. The final step in Brock, Canary and Minsky's proof of the ending lamination conjecture is to show that this model is also bi-Lipschitz. This is done in [BCM04]. On the other hand, Theorem 6.16 completely avoids the work in [BCM04] which seems significant.

\section{References}

[Ag04] I. Agol. Tameness of hyperbolic 3-manifolds. 2004 Preprint.

[Bers60] L. Bers. Simultaneous uniformization. Bull. AMS 66(1960), 94-97.

[Bers70] L. Bers. On boundaries of Teichmüller spaces and on kleinian groups: I. Annals of Math. 91(1970), 570-600.

[Bon86] F. Bonahon. Bouts des variétés hyperboliques de dimension 3. Annals of Math. 124(1986), 71-158.

[Brk00] J. Brock. Continuity of Thurston's length function. Geom. and Funct. Anal. 10(2000), 741-797.

[BB03] J. Brock and K. Bromberg. Grafting, deforming and drilling: cone-manifolds and the density conjecture. In Kleinian Groups and Hyperbolic 3-manifolds. London Math Society Lecture Notes, Cambridge University Press, 2003.

[BB04] J. Brock and K. Bromberg. On the density of geometrically finite Kleinian groups. Acta Math. 192(2004), 33-93.

[BB] J. Brock and K. Bromberg. Geometric inflexibility of hyperbolic 3manifolds. In preparation.

[BCM04] J. Brock, R. Canary, and Y. Minsky. The classification of Kleinian surfaces groups II: the ending lamination conjecture. 2004 Preprint.

[Br02] K. Bromberg. Projective structures with degenerate holonomy and the Bers' density conjecture. 2002 Preprint available at front.math.ucdavis.edu/math.GT/0211402.

[Br04] K. Bromberg. Hyperbolic cone-manifolds, short geodesics and Schwarzian derivatives. J. Amer. Math. Soc. 17(2004), 783-826.

[Br2] K. Bromberg. Rigidity of geometrically finite hyperbolic cone-manifolds. Geom. Dedicata 105(2004), 143-170.

[CG04] D. Calegari and D. Gabai. Shrinkwrapping and the taming of hyperbolic 3-manifolds. 2004 Preprint. 
[CCHS03] R. D. Canary, M. Culler, S. Hersonsky, and P. B. Shalen. Approximation by maximal cusps in the boundaries of quasiconformal deformation spaces. J. Diff. Geom. 64(2003), 57-109.

[CH04] R. D. Canary and S. Hersonsky. Ubiquity of geometric finiteness in boundaries of deformation spaces of hyperbolic 3-manifolds. Amer. J. of Math. 126(2004), 1193-1220.

[Ea80] C. J. Earle. On variation of projective structures. In I. Kra and B. Maskit, editors, Riemann Surfaces and Related Topics: Proceedings of the 1978 Stony Brook Conference, volume 97 of Annals of Math. Studies, pages 87-99. Princeton University Press, 1980.

[Ev03] R. Evans. The ending lamination conjecture for super-slender hyperbolic 3-manifolds. Preprint 2003.

[FG01] L. Funar and S. Gadgil. Topological geodesics and virtual rigidity. Alg. and Geom. Topology 1(2001), 369-380.

[Geh78] F. W. Gehring. Spirals and the universal Teichmüller space. Acta Math. 141(1978), 99-113.

[Hej75] D. A. Hejhal. Monodromy groups and linearly polymorphic functions. Acta Math. 135(1975), 1-55.

[HK98] C. Hodgson and S. Kerckhoff. Rigidity of hyperbolic cone-manifolds and hyperbolic Dehn surgery. J. Diff. Geom. 48(1998), 1-59.

[HK02] C. Hodgson and S. Kerckhoff. Universal bounds for hyperbolic Dehn surgery. 2002 Preprint available at front.math.ucdavis.edu/math.GT/0204345.

[HK03] C. Hodgson and S. Kerckhoff. Harmonic deformations of hyperbolic 3manifolds. In Kleinian Groups and Hyperbolic 3-manifolds. London Math Society Lecture Notes, Cambridge University Press, 2003.

[HK] C. Hodgson and S. Kerckhoff. The shape of hyperbolic Dehn surgery space. In preparation.

[Hub80] J. H. Hubbard. The monodromy of projective structures. In I. Kra and B. Maskit, editors, Riemann Surfaces and Related Topics: Proceedings of the 1978 Stony Brook Conference, volume 97 of Annals of Math. Studies, pages 257-275. Princeton University Press, 1980.

[KS02] G. Kleineidam and J. Souto. Algebraic convergence of function groups. Comment. Math. Helv. 77(2002), 244-269. 
[Mc91] C. McMullen. Cusps are dense. Annals of Math. 133(1991), 217-247.

[Mc96] C. McMullen. Renormalization and 3-Manifolds Which Fiber Over the Circle. Annals of Math. Studies 142, Princeton University Press, 1996.

[Min01] Y. Minsky. Bounded geometry for Kleinian groups. Invent. Math. 146(2001), 143-192.

[Min02] Y. Minsky. The classification of Kleinian surface groups I: models and bounds. 2002 Preprint.

[Osh90] K. Oshika. Ending laminations and boundaries for deformation spaces of Kleinian groups. J. London Math. Soc. 42(1990), 111-121.

[Ot95] J. P. Otal. Sur le nouage des géodésiques dans les variétés hyperboliques. $C$ R. Acad. Sci. Paris 320(1995), 847-852.

[Ot03] J. P. Otal. Les géodésiques fermées d'une variété hyperbolique en tant que noeuds. In Kleinian Groups and Hyperbolic 3-manifolds. London Math Society Lecture Notes, Cambridge University Press, 2003.

[Th79] W. P. Thurston. Geometry and Topology of Three-Manifolds. Princeton lecture notes, 1979.

[Th86] W. P. Thurston. Zippers and univalent functions. In A. Baernstein et al, editor, The Bieberbach conjecture, pages 185-197. AMS, 1986.

[Th] W. P. Thurston. Hyperbolic structures on 3-manifolds II: Surface groups and 3-manifolds which fiber over the circle. Preprint. 
26 Bromberg

K. Bromberg

Department of Mathematics

University of Utah

155 S 1400 E, JWB 233

Salt Lake City, UT 84112

USA

bromberg@math.utah.edu

AMS Classification: 30F40, 37F30, 37F15

Keywords: Kleinian group, hyperbolic 3-manifold, cone-manifold 